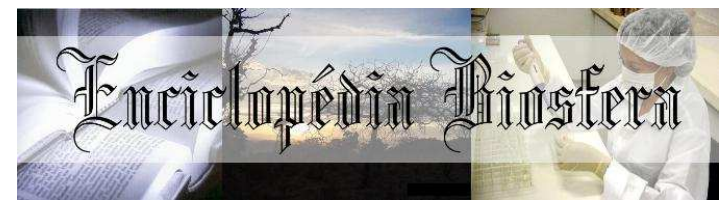

\title{
VIGOR E TEMPO DE ARMAZENAMENTO DE SEMENTES DE SOJA COM TRATAMENTO INDUSTRIAL
}

\author{
Diego Lanferdini ${ }^{1}$, Aline Klug Radke²,Géri Eduardo Meneghello ${ }^{3}$ \\ 1 Mestrando no Programa de Pós Graduação em Ciência e Tecnologia de Sementes \\ da Universidade Federal de Pelotas - Pelotas- Brasil \\ 2 Doutoranda (o) no Programa de Pós Graduação em Ciência e Tecnologia de \\ Sementes da Universidade Federal de Pelotas (alinekradke@hotmail.com) Pelotas- \\ Brasil \\ 3 Professor Doutor do Programa de Pós Graduação em Ciência e Tecnologia de \\ Sementes da Universidade Federal de Pelotas - Pelotas - Brasil \\ Recebido em: 02/10/2017 - Aprovado em: 21/11/2017 - Publicado em: 05/12/2017 \\ DOI: 10.18677/EnciBio_2017B69
}

\begin{abstract}
RESUMO
A performance fisiológica das sementes é afetada por diversos fatores dentre eles o armazenamento, a qualidade inicial do lote e do tratamento químico a que é submetido. Sendo assim, objetivou-se avaliar a qualidade fisiológica de semente de sojas tratadas submetidas ao armazenamento após tratamento industrial. $O$ experimento foi conduzido na Fazenda Sperafico, no município de Sapezal no Mato Grosso. Foram utilizados dois lotes de cada variedade, com qualidades distintas, ou seja, um com alto vigor e outro com médio vigor. As sementes foram tratadas industrialmente, sendo utilizado para tratamento o fungicida Derosal Plus ${ }^{\circledR}$ na dosagem de $200 \mathrm{ml}$ de produto comercial para $100 \mathrm{~kg}$ de sementes e inseticida Standak® na dosagem de $200 \mathrm{ml}$ de produto comercial para $100 \mathrm{~kg}$ de sementes. A avaliação da qualidade fisiológica foi realizada, no dia do tratamento das sementes e após 20, 40 e 60 dias de tratamento. As variáveis analisadas foram: teste de vigor em solo e ambiente controlado e emergência em solo e ambiente controlado. $O$ delineamento experimental utilizado foi inteiramente casualisado, com tratamentos dispostos em fatorial, sendo dois níveis de vigor (alto e baixo) em quatro períodos de avaliação com três repetições por tratamento. Conclui-se que em ambas cultivares, ao se utilizar sementes com alto vigor as mesmas não sofrem decréscimos nos seus níveis de vigor e emergência tanto em ambiente controlado como em solo no período de armazenamento avaliado. Os níveis de vigor das sementes de baixo vigor tratadas, sofrem redução na qualidade fisiológica após 40 dias de armazenamento.
\end{abstract}

PALAVRAS-CHAVE: Glycine max (L.) Merrill, germinação, tratamento.Plântula. 


\title{
INDUSTRIAL TREATMENT OF SEEDS: INFLUENCE OF VIGOR AND TIME OF STORAGE OF SOYBEAN SEEDS
}

\begin{abstract}
The physiological performance of the seeds is affected by several factors, among them the storage, the initial quality of the lot and the chemical treatment to which it is submitted. The objective of this work was to evaluate the quality of seed physiology of treated soybeans submitted to storage after industrial treatment. The experiment was conducted at Fazenda Sperafico, in the municipality of Sapezal, Mato Grosso. Two lots of each variety were used, with distinct qualities, that is, one with high vigor and another with medium vigor. The seeds were treated industrially and the fungicide Derosal Plus $\AA$ was used in the dosage of $200 \mathrm{ml}$ of commercial product for $100 \mathrm{~kg}$

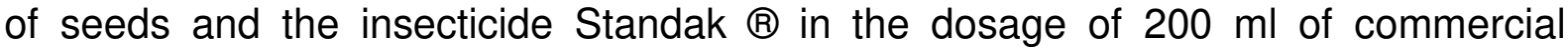
product for $100 \mathrm{~kg}$ of seeds. The evaluation of physiological quality was performed on the day of seed treatment and after 20, 40 and 60 days of treatment. The variables analyzed were: vigor test in soil and controlled environment and emergence in soil and controlled environment. The experimental design was completely randomized, with treatments arranged in factorial, with two levels of vigor (high and low) in four evaluation periods with three replications per treatment.. It is concluded that in both cultivars, when using seeds with high vigor they do not suffer decreases in their levels of vigor and emergence both in controlled environment and in soil in the period of storage evaluated. The vigor levels of the treated low vigor seeds are reduced in physiological quality after 40 days of storage.
\end{abstract}

KEYWORDS: Glycine max (L.) Merrill, seedling, germination, treatment

\section{INTRODUÇÃO}

A soja, Glycine max (L.) Merrill é uma das culturas de maior importância mundial, e a mais importante do Brasil. O grão pode ser amplamente utilizado para a elaboração de rações animais, produção de óleo e outros subprodutos, além do seu consumo in natura que segundo Araújo (2009) vem se expandindo nas últimas décadas, fato este que é observado até os dias atuais (CONAB, 2017).

O tratamento de sementes é uma tecnologia que, quando associada a melhoramento genético vegetal, biotecnologia e demais recomendações técnicas, permite alta produtividade da cultura da soja e satisfação do produtor em atender às demandas do mercado. Este mercado, por sua vez, é apoiado pelo uso de sementes de qualidade que, como veículo de tecnologia e inovações (FERREIRA et al., 2016).

Além dos aspectos elencados, é importante salientar que o tratamento de sementes é uma prática agrícola preventiva, que consiste na aplicação de fungicidas e/ou inseticidas na superfície da semente (CONCEIÇÃO et al., 2014), visando não só controlar os patógenos durante o armazenamento, mas igualmente proteger as plântulas durante a germinação e no período inicial de instalação da lavoura, ou seja, de emergência. Na maioria dos países em que a agricultura é intensiva e altamente produtiva, o tratamento é realizado, basicamente nas próprias unidades de beneficiamento de sementes, de forma industrial, forma de tratamento que tem sido largamente utilizado no Brasil nos últimos anos.

Quando a semente é utilizada, na semeadura de uma nova safra, já passou por um período de armazenamento, logo é prática comum na agroindústria de sementes da cultura realizar o tratamento industrial de sementes com no máximo 60 dias de antecedência do início da semeadura no intuito de minimizar os possíveis efeitos nocivos das caldas utilizadas neste processo (ZAMBAN, 2013). 
O armazenamento correto das sementes até o momento de sua utilização é uma etapa importante do processo de produção de sementes de alta qualidade, uma vez que o armazenamento não melhora a qualidade, somente a mantém por um período variável de tempo. Durante o período de armazenamento, o tratamento das sementes com fungicidas pode favorecer a manutenção da qualidade fisiológica e o aumento da vida útil das sementes, além de melhorar a qualidade sanitária (PEREIRA et al., 2007).

Para o tratamento de sementes ser efetivo é preciso que os produtos proporcionem uma adequada cobertura sobre toda a superfície da semente, minimizando com isso a ação dos patógenos. Por outro lado, é necessário que os produtos não prejudiquem o desempenho das sementes, mantendo, se possível, inalterada a sua qualidade fisiológica. Por este motivo, pesquisar a influência desses produtos aplicados às sementes durante 0 armazenamento, particularmente no ambiente de armazenamento, possibilitará garantir a comercialização das sementes de soja com alta qualidade fisiológica (PEREIRA et al., 2016), ou seja, a indústria sementeira precisa ajustar a logística de tratamento de forma que este seja feito em um momento que estes dois processos ocorram, porém não interferindo na qualidade fisiológica das sementes. Objetivou-se com o presente trabalho avaliar os efeitos do tratamento de sementes na qualidade fisiológica das sementes, em lotes de qualidade distintas, durante $o$ armazenamento.

\section{MATERIAL E MÉTODOS}

O experimento foi realizado no Laboratório de Análise de Sementes, da Fazenda Sperafico, pertencente ao Grupo Scheffer, localizada em Sapezal - MT. Os experimentos foram conduzidos com sementes, das variedades M 7739IPRO precoce, grupo de maturação 7.7, habito de crescimento semi-determinado e AS 3820IPRO precoce, grupo de maturação 8.2, hábito de crescimento determinado.

Foram utilizados dois lotes de cada variedade, com qualidades distintas, ou seja, um com alto vigor e outro com médio vigor. As sementes foram tratadas industrialmente em uma máquina de tratamento industrial de sementes (TSI) de fluxo continuo da marca Momesso, modelo Seedmix VHS 10t. Sendo utilizado para tratamento o fungicida Derosal Plus ${ }^{\circledR}$ na dosagem de $200 \mathrm{ml}$ de produto comercial para $100 \mathrm{~kg}$ de sementes e inseticida Standak ${ }^{\circledR}$ na dosagem de $200 \mathrm{~mL}$ de produto comercial para $100 \mathrm{~kg}$ de sementes.

Após o tratamento as sementes ficaram armazenadas no armazém de sementes da unidade, com temperatura média $20 \pm^{\circ} \mathrm{C}$ e umidade relativa de $50 \%$, acondicionadas em sacos de papel multifoliado sob pallet de madeira.

A avaliação da qualidade fisiológica foi realizada, no dia do tratamento das sementes e após 20,40 e 60 dias de tratamento. Para a realização dos testes foi coletada uma amostra de cada lote por repetição, submetidas a homogeneização e a partir dessa amostra extraída a quantia para semeadura de três repetições por lote de cada variedade, em cada uma das épocas. Foram realizadas as seguintes determinações:

\section{Teste de vigor em ambiente controlado (VAC)}

Foram utilizadas quatro sub amostras de 50 sementes, distribuídas na superfície de uma camada de $5 \mathrm{~cm}$ de areia colocada em caixas de plástico $(32 \mathrm{~cm} \times$ $28 \mathrm{~cm} \times 10 \mathrm{~cm}$ ). Após a semeadura, a cobertura foi efetuada com uma camada de 3 
$\mathrm{cm}$ de areia. $\mathrm{O}$ umedecimento do substrato foi efetuado com quantidade de água correspondente a $60 \%$ da capacidade de retenção. As caixas foram mantidas em estufa climatizadas a temperatura de $25^{\circ} \mathrm{C}$ e UR $50 \%$, com 12 horas de luz e 12 horas de escuro. No sexto dia após a semeadura foi realizada a contagem de plântulas normais emersas, determinando a porcentagem de vigor. $O$ vigor foi determinado quando a plântula apresenta todas as estruturas fisiológicas em perfeito estado sendo elas os cotilédones, plúmula, sistema radicular e hipocótilo. Considerou-se plântula vigorosa aquela que teve seu desenvolvimento inicial rápido. O resultado foi expresso em porcentagem.

\section{Teste de Emergência em Ambiente Controlado (EAC)}

Realizado em conjunto com o VAC, porém foram avaliadas as plântulas que apresentaram algum tipo de dano seja por: umidade, picada de percevejo ou dano mecânico, que não afetaram sua reserva ou seu embrião, mas que deterioraram uma parte significativa de sua estrutura dificultando assim seu desenvolvimento. Também foram avaliadas as plântulas anormais (AAC), determinou-se como padrão de avaliação as sementes ou plântulas germinadas que apresentaram um ou mais danos que impossibilitaram seu desenvolvimento. O resultado foi expresso em porcentagem.

\section{Teste de emergência em solo (ES)}

Foram utilizadas duas sub amostras de 100 sementes, semeadas em linhas, 5 linhas espaçadas $5 \mathrm{~cm}$, cada uma com 20 sementes, na profundidade de $3 \mathrm{~cm}$. Os canteiros foram preparados no dia anterior a semeadura, afim de manter a umidade ideal para a semeadura e molhados diariamente ao final da tarde. Foi analisada a parte aérea e analisado os sistemas radiculares com a retirada das plantas do canteiro. Assim foi possível a separação de plantas normais e anormais que possuem um ou mais tipos de danos. Considerou-se como padrão plântulas normais as que possuem danos menores de $2 \%$ das áreas cotiledonares de umidade ou picada de percevejo que não seja na reserva ou no embrião e o restante das estruturas estejam em perfeito estado, o restante foi considerado plântulas anormais (AS). As avaliações foram realizadas no sétimo e décimo segundo dia após a semeadura, avaliando-se plântulas normais e anormais.

\section{Teste de vigor em solo (VS)}

Conduzido juntamente com o teste de germinação, sendo a contagem realizada no sétimo dia após a semeadura. Nesta avaliação foram analisadas as plântulas que apresentaram sua estrutura aérea em perfeito estado sendo eles: cotilédones, plúmula e hipocótilo.

\section{Delineamento estatístico}

O delineamento experimental utilizado foi o inteiramente casualizado, com tratamentos dispostos em fatorial $(2 \times 4)$, sendo dois níveis de vigor (alto e baixo) e quatro períodos de avaliação (0,20, 40 e 60 dias após o tratamento de sementes), com três repetições. Os dados foram submetidos a análise de variância, e posteriormente, havendo significância para a interação entre os fatores, foram realizados os devidos desdobramento, seguido de comparação de médias para o fator níveis de vigor e regressão polinomial para o fator tempo de armazenamento. 


\section{RESULTADOS E DISCUSSÃO}

Observa-se na tabela 1, que em ambas cultivares o lote de alto vigor foi superior ao lote de baixo vigor nos testes de vigor e germinação em areia e nos testes de vigor e germinação em canteiro no período avaliado, no entanto no período de 40 dias no teste de vigor e germinação em canteiro não houve diferenciação no número de plantas anormais.

É importante salientar que sementes de soja armazenadas sob mesma condição podem apresentar variação no tempo que levam para perder seu potencial fisiológico, pois estão relacionadas a qualidade inicial da semente, sendo que lotes com maior qualidade inicial mantêm a qualidade durante o armazenamento e possuem maior tolerância a deterioração (CARVALHO; NAKAGAWA, 2000).

TABELA 1 - Percentagem de emergência, vigor, e plântulas anormais em ambiente controlado e solo para as duas cultivares em função de distintos níveis de vigor e armazenamento após tratamento químico das sementes.

\begin{tabular}{|c|c|c|c|c|c|c|c|}
\hline Etapa & Lote & VAC & EAC & ACC & VS & ES & AS \\
\hline \multicolumn{8}{|c|}{ Cultivar M 7739 IPRO } \\
\hline \multirow{2}{*}{0} & Baixo Vigor & $70 \mathrm{~b}$ & $80 \mathrm{~b}$ & $14 \mathrm{a}$ & $63 \mathrm{~b}$ & $76 \mathrm{~b}$ & $10 \mathrm{a}$ \\
\hline & Alto Vigor & $90 \mathrm{a}$ & $94 \mathrm{a}$ & $4 \mathrm{~b}$ & $82 \mathrm{a}$ & $95 \mathrm{a}$ & $2 b$ \\
\hline \multirow{2}{*}{20} & Baixo Vigor & $71 \mathrm{~b}$ & $79 \mathrm{~b}$ & $18 a$ & $62 \mathrm{~b}$ & $78 \mathrm{~b}$ & $8 \mathrm{a}$ \\
\hline & Alto Vigor & $91 \mathrm{a}$ & $94 \mathrm{a}$ & $5 b$ & $85 a$ & $95 \mathrm{a}$ & $2 \mathrm{~b}$ \\
\hline \multirow{2}{*}{40} & Baixo Vigor & $65 \mathrm{~b}$ & $78 \mathrm{~b}$ & $16 \mathrm{a}$ & $50 \mathrm{~b}$ & $67 \mathrm{~b}$ & $4 \mathrm{a}$ \\
\hline & Alto Vigor & $89 \mathrm{a}$ & $96 \mathrm{a}$ & $2 b$ & $84 \mathrm{a}$ & $90 \mathrm{a}$ & $4 \mathrm{a}$ \\
\hline \multirow{2}{*}{60} & Baixo Vigor & $57 \mathrm{~b}$ & $64 \mathrm{~b}$ & $20 \mathrm{a}$ & $48 \mathrm{~b}$ & $60 \mathrm{~b}$ & $8 \mathrm{a}$ \\
\hline & Alto Vigor & $87 \mathrm{a}$ & $93 \mathrm{a}$ & $4 \mathrm{~b}$ & $83 a$ & $92 \mathrm{a}$ & $3 \mathrm{~b}$ \\
\hline C.V.(\%) & & 5,2 & 5,5 & 26,8 & 5,2 & 3,4 & 23,0 \\
\hline \multicolumn{8}{|c|}{ Cultivar AS 3820 IPRO } \\
\hline \multirow{2}{*}{0} & Baixo Vigor & $72 \mathrm{~b}$ & $79 \mathrm{~b}$ & $16 \mathrm{a}$ & $63 \mathrm{~b}$ & $74 \mathrm{~b}$ & $18 \mathrm{a}$ \\
\hline & Alto Vigor & $84 \mathrm{a}$ & $89 a$ & $7 \mathrm{~b}$ & $77 \mathrm{a}$ & $91 \mathrm{a}$ & $5 b$ \\
\hline \multirow{2}{*}{20} & Baixo Vigor & $71 \mathrm{~b}$ & $81 \mathrm{~b}$ & $15 a$ & $54 \mathrm{~b}$ & $78 \mathrm{~b}$ & $9 a$ \\
\hline & Alto Vigor & $84 a$ & $89 a$ & $8 b$ & $79 a$ & $88 \mathrm{a}$ & $3 b$ \\
\hline \multirow{2}{*}{40} & Baixo Vigor & $71 \mathrm{~b}$ & $78 \mathrm{~b}$ & $13 \mathrm{a}$ & $49 \mathrm{~b}$ & $66 \mathrm{~b}$ & $5 a$ \\
\hline & Alto Vigor & $82 \mathrm{a}$ & $89 \mathrm{a}$ & $8 \mathrm{~b}$ & $72 \mathrm{a}$ & $87 \mathrm{a}$ & $4 \mathrm{a}$ \\
\hline \multirow{2}{*}{60} & Baixo Vigor & $57 \mathrm{~b}$ & $69 \mathrm{~b}$ & $21 \mathrm{a}$ & $38 \mathrm{~b}$ & $60 \mathrm{~b}$ & $17 \mathrm{a}$ \\
\hline & Alto Vigor & $82 \mathrm{a}$ & $89 \mathrm{a}$ & $8 \mathrm{~b}$ & $74 \mathrm{a}$ & $86 a$ & $4 \mathrm{~b}$ \\
\hline C.V.(\%) & & 3,2 & 3,3 & 17,5 & 6,8 & 4,7 & 18,5 \\
\hline
\end{tabular}

${ }^{*}$ Medias seguidas de mesma letra na coluna, em cada etapa, não diferem entre si pelo teste de tukey a $5 \%$ de probabilidade de erro.

Pode-se observar, para a cultivar M 7739 IPRO, lote de alto e baixo vigor, as variáveis vigor e emergência (Figura $1 \mathrm{~A}$ e 1B) em ambiente controlado, vigor e emergência em solo (Figura 1C e 1D) houve redução linear durante o período de armazenamento, sendo mais acentuado a partir dos 40 dias após o tratamento. Segundo Brzezinski et al. (2015), o tratamento de sementes pode inferir algumas limitações no desenvolvimento, tais como os possíveis efeitos dos ingredientes ativos na qualidade de as sementes durante o armazenamento e no campo.

Destaca-se, no entanto que o lote de alto vigor não teve decréscimo nos testes realizados durante o período de armazenamento, significando que estas sementes mobilizam suas reservas de formas mais ordenada, superando as 
condições adversas do ambiente. As pequenas variações observadas nos lotes de alto vigor, fez com que, em algumas variáveis respostas, não fosse possível ajustar em um modelo polinomial significativo.

Os resultados encontrados estão de acordo com o observado por Ludwig et al. (2014), que concluíram, quando pesquisaram o efeito do tratamento químico das sementes, que não há efeito negativo logo após o tratamento das sementes sobre o desempenho inicial das plântulas. De forma semelhante, Dan et al. (2011) observaram que o tratamento com inseticidas, em sementes de soja, promove adequados níveis de germinação e vigor, durante o período de armazenamento sete dias.
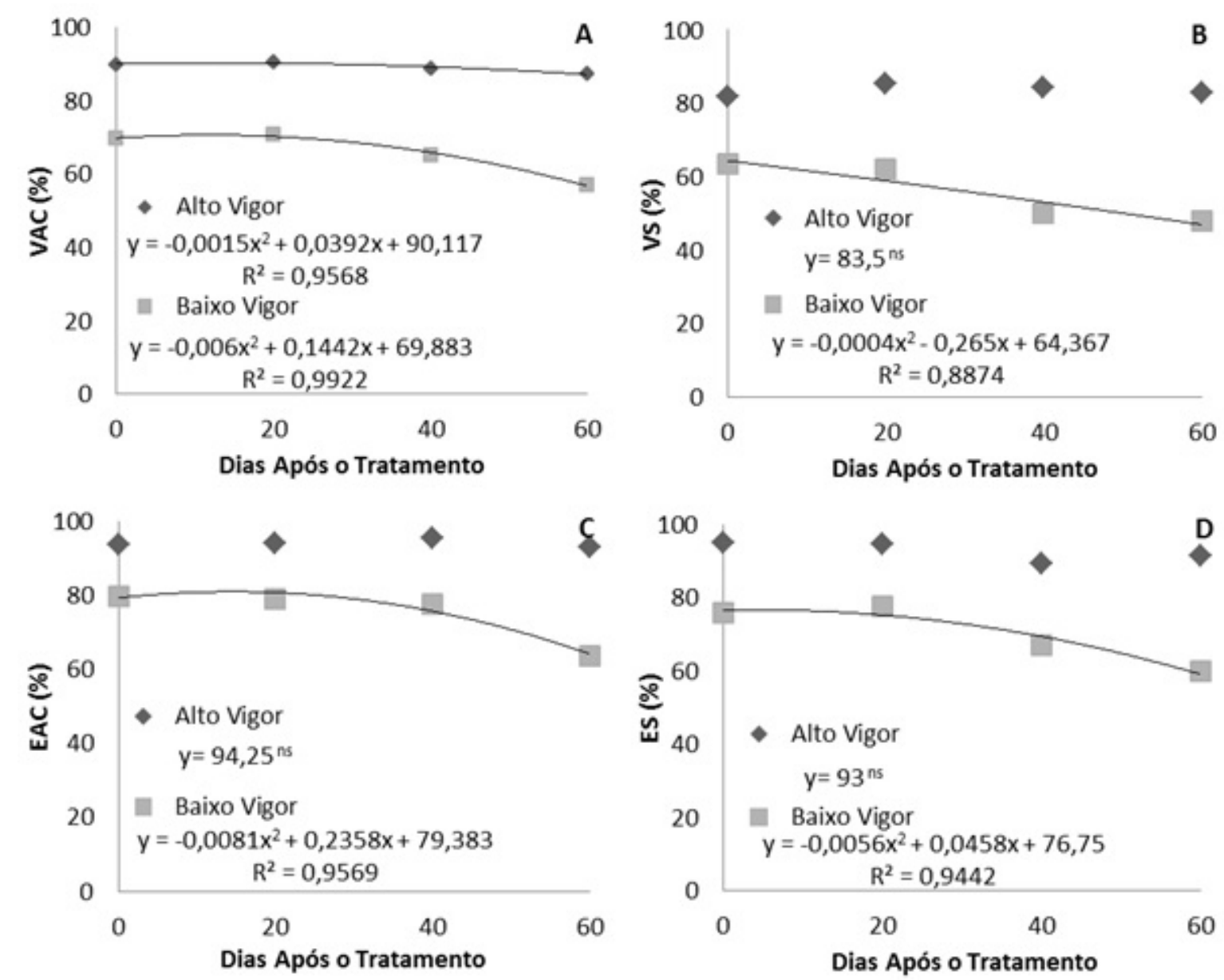

FIGURA 1 - Percentagem plântulas normais no teste vigor em ambiente controlado (A) e solo (B), teste de emergência em ambiente controlado (C) e solo (D), para a cultivar M7739 PRO em função de distintos níveis de vigor e armazenamento após tratamento químico das sementes.

$\mathrm{Na}$ figura 2, observa-se que para todos os testes realizados a cultivar AS 3820 IPRO não apresentou resultado significativo para o lote de alto vigor, porém este resultado mostra-se adequado, visto que sementes de alto vigor resistem a condições adversas de ambiente. Tavares et al. (2013) destacam que plantas oriundas de sementes de alto vigor apresentam maior taxa de crescimento inicial. 
Quando avaliadas as sementes de baixo vigor as mesmas sofreram redução nos em seus níveis de qualidade (Figura 2A e 2B) de forma acentuada após os 40 dias de armazenamento. Dan et al. (2010), afirmam que ocorre redução da qualidade fisiológica das sementes, condicionada pelo tratamento químico, e acaba intensificando com o prolongamento do período de armazenamento. Além disso, sementes com baixa qualidade fisiológica comprometem a obtenção de um adequado estande de plantas no campo, influindo diretamente na produtividade da lavoura (FRANDOLOSO et al., 2015).
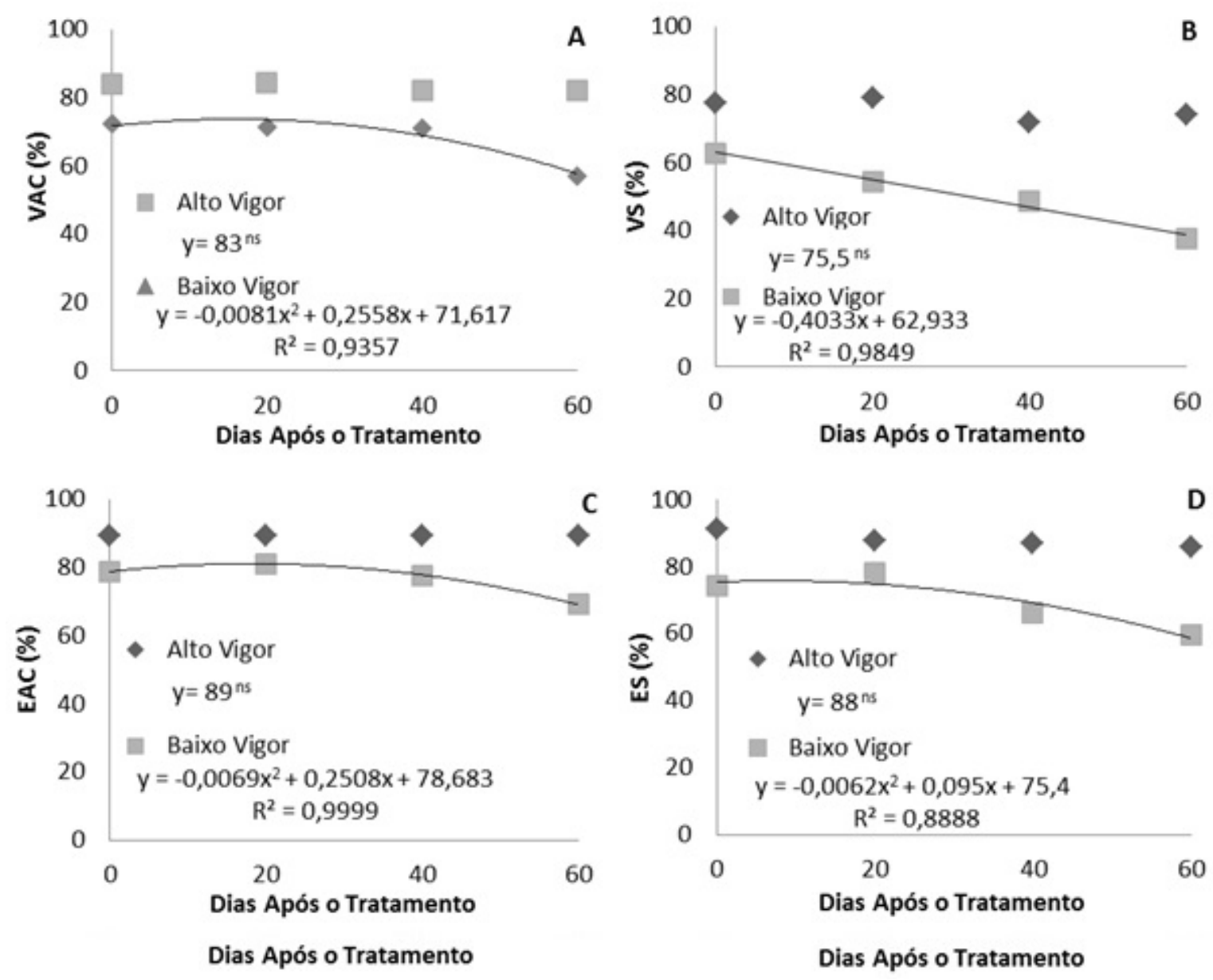

FIGURA 2 - Percentagem plântulas normais no teste de vigor em ambiente controlado $(A)$ e solo $(B)$, teste de emergência em ambiente controlado (C) e solo (D), para a cultivar AS3820 IPRO em função de distintos níveis de vigor e armazenamento após tratamento químico das sementes.

Com base nos resultados obtidos, é possível observar a importância da escolha adequada do tempo de armazenamento e a escolha por sementes vigoras. As cultivares utilizadas neste estudo podem ser consideradas contrastantes entre si. A cultivar Agroeste AS 3820 IPRO, grupo de Maturação 8.2, 112 a 118 dias até a colheita e hábito de crescimento determinado. Por outro lado, M 7739IPRO é uma cultivar Monsoy, grupo de maturação 7.7, (102 a 118) dias até a colheita e hábito de 
crescimento semi indeterminado. Considerando que houve semelhança entre ambas no armazenamento, aspectos de qualidade fisiológica inicial das sementes preponderaram sobre a questão genética.

\section{CONCLUSÃO}

Em ambas cultivares, ao utilizar sementes com alto vigor as mesmas não sofrem decréscimos nos níveis de vigor e emergência tanto em ambiente controlado como em solo no período de armazenamento avaliado.

Os níveis de vigor das sementes de baixo vigor tratadas, sofrem redução na qualidade fisiológica após 40 dias de armazenamento.

\section{REFERÊNCIAS}

ARAÚJO, M. M. Caracterização e seleção de linhagens de soja resistentes ou tolerantes à ferrugem asiática. Piracicaba: ESALQ, 2009. 77p.

BRZEZINSKI, C. C.; HENNING, A. A.; ABATI, J.; HENNING, F. A.; FRANÇA-NETO, J. B.; et al. Seeds treatment times in the establishment and yield performance of soybean crops. Journal of Seed Science, v.37, n.2, p.147-153, 2015. Disponível em: <http://www.scielo.br/pdf/jss/v37n2/2317-1537-jss-37-02-00147.pdf>.

DOI: $10.1590 / 2317-1545 v 37 n 2148363$

CARVALHO, N. M.; NAKAGAWA, J. Sementes: ciência, tecnologia e produção. 4. ed. Jaboticabal: FUNEP, 2000.

CONAB - Companhia Nacional de Abastecimento. Disponível em <http:/www.conab.gov.br> Acesso em: 14 junho de 2017.

CONCEIÇÃO, G.M.; BARBIERI, A.P.P.; LÚCIO, A.D.; MARTIN, T.N.; MERTZ, L.M.; et al. Desempenho de plântulas e produtividade de soja submetida a diferentes tratamentos químicos nas sementes. Bioscience Journal, v. 30, n. 6, p. 1711-1720, 2014.

Disponível em:

http://www.seer.ufu.br/index.php/biosciencejournal/article/view/22024/15608>.

DAN, L. G. M.; DAN, H. A.; BARROSO, A. L. L.; BRACCINI, A. L. Qualidade fisiológica de sementes de soja tratadas com inseticidas sob efeito do armazenamento. Revista Brasileira de Sementes, Londrina. v. 32, n. 2 p. 131-139, 2010. Disponível em: < http://www.scielo.br/pdf/rbs/v32n2/v32n2a16.pdf>.

DAN, L.G.M.; DAN, H.A.; BRACCINI, A.L.; ALBRECHT, L.P.; RICCI, T.T.; PICCININ, G.G. Desempenho de sementes de soja tratadas com inseticidas e submetidas a diferentes períodos de armazenamento. Revista Brasileira de Ciências Agrárias, v.6. $\quad$ n. 2, p.215-222, 2011. Disponível em: < http://www.agraria.pro.br/sistema/index.php?journal=agraria\&page=article \&op=viewA rticle\&path\%5B\%5D=agraria_v6i2a939>. DOI: 10.5039/agraria.v6i2a939

FERREIRA, T.F.; OLIVEIRA, J.A.; CARVALHO, R.A.; RESENDE, L.S.; LOPES, C.G.M.; et al. Quality of soybean seeds treated with fungicides and insecticides before and after storage. Journal of Seed Science, v.38, n.4, p.278-286, 2016. 
Disponível em: <http://www.scielo.br/pdf/jss/v38n4/2317-1545-jss-38-04-00278.pdf>. DOI: 10.1590/2317-1545v38n4161760

FRANDOLOSO, V.; MENEGHELLO, G.E.; SUAREZ, C.I.; TILLMANN, A.A.; DEUNER. Qualidade física e sanitária de sementes de soja produzidas no estado de Santa Catarina. Semina: Ciências Agrárias, v. 36, n. 4, p. 2515-2526, 2015. Disponível em:

http://www.uel.br/revistas/uel/index.php/semagrarias/article/view/18092/16780>. DOI: 10.5433/1679-0359.2015v36n4p2515

LUDWIG, E. J.; NUNES, U. R.; MERTZ, L. M.; SILVA, J. R. da; NUNES, S. C. P. Vigor e produção de sementes de crambe tratadas com fungicida, inseticida e polímero. Científica, v.42, n.3, p.271-277, 2014.

PEREIRA, C.E.; OLIVEIRA, J.A.; EVANGELISTA, J.R.E.; BOTELHO, F.J.E.; OLIVEIRA, G.E.; TRENTINI, P. Desempenho de sementes de soja tratadas com fungicidas e peliculizadas durante 0 armazenamento. Ciência e Agrotecnologia, v.31, n.3, p.656-665, 2007. Disponível em: < http://www.scielo.br/scielo.php?script=sci_arttext\&pid=S1413-70542007000300009>. DOI: 10.1590/S1413-70542007000300009

PEREIRA, L. C.; GARCIA, M. M.; BRACCINI, A. L.; PIANA, S. C.; CRISTINA, G.; et al. Efeito da adição de biorregulador ao tratamento industrial sobre a qualidade de sementes de soja (Glycine max (L.) Merr.) aos sessenta dias de armazenamento convencional. Revista colombiana de investigaciones agroindustriales, v. 3, p. 15-22, 2016. Disponível em: <https://doi.org/10.23850/24220582.347>. DOl: $10.23850 / 24220582.347$

TAVARES, L.C.; RUFINO, C.A.; BRUNES, A.P.; TUNES, L.M.; BARROS, A.C.S.A.; PESKE, S.T. I. Desempenho de sementes de soja sob deficiência hídrica: rendimento e qualidade fisiológica da geração F1. Ciência Rural, v.43, n.8, p.13571363, 2013. Disponível em: <http://dx.doi.org/10.1590/S0103-84782013000800003>. DOI: 10.1590/S0103-84782013000800003

ZAMBAN, S. Aspectos importantes do Tratamento de Sementes. Informativo Abrates, v. 23, n.2, p. 26-32, 2013. 